\title{
ENHANCING EXPLANATION COHERENCE WITH RHETORICAL STRATEGIES
}

\author{
MARK T. MAYBURY \\ Rome Air Development Center \\ Intelligent Interface Group \\ Griffiss AFB, Rome NY 13441-5700 \\ maybury@radc-tops20.arpa \\ and \\ Cambridge University Computer Laboratory \\ Cambridge, England CB2 3QG
}

\begin{abstract}
This paper discusses the application of a previously reported theory of explanation rhetoric (Maybury, 1988b) to the task of explaining constraint violations in a hybrid rule/frame based system for resource allocation (Dawson et al, 1987). This research illustrates how discourse strategies of explanation, textual connectives, and additional justification knowledge can be applied to enhance the cohesiveness, structure, and clarity of knowledge based system explanations.
\end{abstract}

\section{INTRODUCTION}

Recent work in text generation includes emphasis on producing textual presentations of the explanations of reasoning in knowledge-based systems. Initial work (Swartout, 1981) on the direct translation of underlying system knowledge led to insights that more perspicuous justifications would result from keeping track of the principles or deep causal models which supported that knowledge (Swartout and Smoliar, 1988). And experiments with discourse strategies demonstrated the efficacy of the rhetorical organization of knowledge to produce descriptions, comparisons (McKeown, 1985) and clarification (McCoy, 1985). Researchers have recently observed (Paris et al, 1988) that the line of explanation should not isomorphically mirror the underlying line of reasoning as this often resulted in poorly connected text (Appelt, 1982). Others have attempted to classify patterns of explanations
(Stevens and Steinberg, 1981; Schank, 1986). The approach presented here is to exploit generic explanation strategies and focus models (Sidner, 1983; Grosz and Sidner, 1988) to organize the back-end justification via an explanation rhetoric -- that is, a rhetorical model of strategies that humans employ to persuade, support, or clarify their position. The result is a more connected, flowing and thus easier to follow textual presentation of the explanation.

\section{KNOWLEDGE REPRESENTATION and EXPLANATION}

Previous research in natural language generation from knowledge based systems has primarily focused on independent knowledge representation schemes (e.g rule, frame or conceptual dependency formalisms). In contrast, the application chosen to test the concepts of rhetorical explanations is an FRL (Roberts and Goldstein, 1977) based mission planning system for the Air Force which utilizes both rules and frames during decision-making. Hence, the explanations concern rule-based constraint violations which result from inference about entities in the knowledge base, their attributes, and relationships. For example, if the user plans an offensive counter air mission with an incompatible aircraft and target, the system will automatically signal a constraint violation via highlighting of objects on the screen. If the user mouses for explanation, the system will state the conflicting rule, then list the supporting knowledge, as shown in figure 1. 
The choice for AIRCRAFT is in question because:

BY TARGET-AIRCRAFT-1:1 THERE IS A SEVERE CONFLICT BETWEEN

TARGET AND AIRCRAFT FOR OCA1002²

\author{
1. THE TARGET OF OCA1002 IS BE30703 3 \\ 2. BE30703 RADIATES \\ 3. THE AIRCRAFT OF OCA1002 IS F-111E \\ 4. F-111E IS NOT A F-4G
}

Figure 1. Current Explanation of Rule Violation

The weak textuality of the presentation manifests itself through ungrammatical sentences and the implicit suggestion of relationships among entities, placing the burden of organization upon the reader. Moreover, it lacks essential content that specifies why an F-111E is not acceptable. That "F-111E IS NOT A F-4G" makes little contribution to the justification, and at best implicitly suggests an alternative (an F-4G). generated with templates followed by a direct translation of the explanation audit trail (a trace of the inferences of the constraint propagation algorithm as shown in figure 2).

The explanation trace is of the form: (rule-constraint (justification-knowledge-type ((justification-content) (support-code)) $\left.)^{*}\right)^{*}$ where ${ }^{*}$ means 1 to $\mathrm{N}$ repetitions. In the example, the rule constraint is TARGET-

((TARGET-AIRCRAFT-1

$\begin{array}{ll}\text { (DATA } & \text { (TARGET OCA1002 BE30703)) } \\ \text { (INHERITANCE } & \text { (IS-A BE30703 ELECTRONICS)) } \\ \text { (DATA } & \text { (AIRCRAFT OCA1002 F-111E) } \\ & \text { ((NOTEQ F-111E (QUOTE F-4G)))))) }\end{array}$

Figure 2. Audit Trail of Constraint Failure

One reason the text lacks coherence is because it fails to specify precise relationships among introduced entities. This can be achieved not only by sequential order, but through the use of models of rhetoric, textual connectives, and discourse devices such as anaphora and pronominal modifiers. For instance, rather than achieving organization from some model of naturally occurring discourse, the presentation is isomorphic to the underlying inference chain. In figure 1, the first two sentences are

\footnotetext{
${ }^{1}$ This is the name of the rule.

${ }^{2}$ Reads "Offensive Counter Air Mission 1002".

${ }^{3}$ Reads "Battle Element number 30703".
}

\begin{abstract}
AIRCRAFT-1, and the two justification types are DATA and INHERITANCE, representing knowledge and relationships among entities in the FRL knowledge base. Notice that the (AIRCRAFT OCA1002 F-111E) tuple is followed by a lisp code test for inequality of F-111E and F-4G aircraft. It is unclear (indeed unspecified) in this formalism that the reason for this test and the preference for an F-4G is its ability to handle search radar. Thus, discrimination of the two aircraft on the basis of structure, function, capability or some other characteristic would further clarify the explanation. Therefor, there is a need not only for linguistic processing to enhance the coherence of the presentation in figure 1 , but also additional knowledge to enhance the perspicuity of the explanation.
\end{abstract}




\section{EXPLANATION RHETORIC}

The implemented system, EXPLAN, exploits models of rhetorical strategies, focus models, as well as entity-distinguishing knowledge to improve the organization, connectivity and surface choices (e.g. connectives and anaphor) of the text. The system first instantiates a pool of relevant explanation propositions from both the explanation audit trail as well as from the knowledge base as both are sources of valuable clarifying information. The text planner uses a predicate selection algorithm (guided by a global and local focus model, knowledge of rhetorical ordering, relationships among entities in the knowledge base, and the explanation audit trail) to select and order propositions which are then realized via a case semantics, a relational grammar, and finally morphological synthesis algorithms (Maybury, 1988a).

In our example, the first task is to determine the salience of entities to the explanation. The generator includes the current frame (that is, the current mission being planned, OCA1002) in the global focus of attention. However, global focus also must include those slots which may have relevance to constraint violations. Figure 3 shows the OCA1002 mission frame which has many slots, only a few of which are central to the explanation, namely the AIRCRAFT and TARGET slots. A selection algorithm filters out semantically irrelevant slots (e.g. AIO, DISPLAY) and retains slots trapped by the constraint violation. Salient objects in the knowledge base are marked, including the parent and children of the object(s) in question (which are explicitly in focus) and the siblings or cousins of the global focus (which are implicitly in focus).

After selecting the global focus (OCA1002, AIRCRAFT, and TARGET), and marking salient objects in the knowledge base, the planner selects three propositions from the instantiated pool guided by the local focus model and the model of explanation discourse. The proposition pool includes previously reported (McKeown, 1985) rhetorical types such as attributive, constituent, and illustration, but also includes a wide range of justificatory rhetorical predicate types such as characteristic, componential, classificatory, physical-causal, generalization, associative, and functional, as reported in (Maybury, 1988b).

These predicates are grouped into subschema as to whether they identify the problem, support the identification or diagnosis, or recommend actions. These sub-strategies, which provide global rhetorical coherence, can expand to a range of predicate types such as the three chosen in the example plan. As figure 4 illustrates, the explanation strategy is a representation of

\begin{tabular}{|lll|}
\hline $\begin{array}{l}\text { (OCA1002 } \\
\text { (AIO }\end{array}$ & & \\
(AUX & (VALUE & $($ OCA))) \\
(DISPLAY & (VALUE & $($ OCA1002-AUX))) \\
(AIRCRAFT & (VALUE & $($ (\#MISSION-WINDOW 1 1142344 deexposed $>)))$ \\
& (POSSIBLE & $($ (F-4C F-4D F-4E F-4G F-111E F-111F))) \\
& (VALUE & $($ F-111E)) \\
& (STATUS & $($ USER))) \\
(HISTORY & (VALUE & $(<\#$ EVENT INSERT TARGET BE30703 USER>))) \\
(AIRBASE & (POSSIBLE & $(($ ALCONBURY))) \\
(ORDNANCE & (POSSIBLE & $(($ A1 A2 ... A14)))) \\
(TARGET & (VALUE & $($ BE30703)) \\
& (STATUS & $($ (USER))) \\
(ACNUMBER & (POSSIBLE & $((12 \ldots 25)))$ \\
& (VALUE & $(3))$ \\
& (STATUS & $($ USER)))) \\
& & \\
& &
\end{tabular}

Figure 3. Mission Frame in FRL 


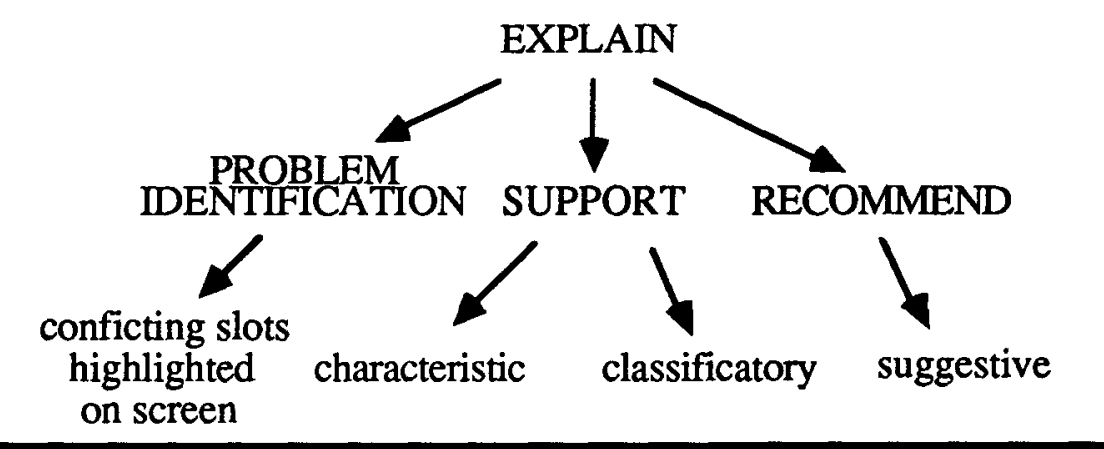

Figure 4. Dominance (arrows) and Ordering (sequential equilevel nodes) relationships

both dominance and ordering among the predicates as well as a means for powerful aggregation of predicates into substrategies. distinguishes between the two fighter entities indicating the deeper reason why the choice is recommended. This knowledge originates

\author{
(CHARACTERISTIC \\ ((OCA1001)) \\ ((AIRCRAFT F-111E) (TARGET NIL NIL BE30703)))
}

(CLASSIFICATORY

((LUDWIGSLUSTS-ALPHA))

((ELECTRONICS NIL NIL NIL NIL NIL ((FUNCTION (EW-GCI))))))

\title{
(SUGGESTIVE
}

((AIRCRAFT SELECTED))

((F-4G NIL NIL NIL NIL NIL ((FUNCTION (RADAR-DESTRUCTION))))

(F-111E NIL NIL NIL NIL NIL ((FUNCTION (RADAR-SUPPRESSION))))))

Figure 5. Selected Rhetorical Propositions.

The corresponding instantiated rhetorical propositions are shown in figure 5 . The problem to be identified in our illustration is that there is a conflict between the aircraft and the target chosen in the mission plan. As this is indicated by highlighting of these slots on the screen, identification of the conflict is not included in the text, although there is no reason why this could not be explicitly stated by means of a definition predicate. With the problem identified, the planner justifies this identification by characterizing the mission under consideration and classifying the object at the root of the constraint violation. Finally, the planner recommends a viable alternative using a suggestive proposition.

Notice that the discriminatory knowledge in the suggestive predicate in figure 5 from the knowledge base ${ }^{1}$ rather than the explanation trace. Thus the knowledge provided in the audit trail along with general knowledge from the domain knowledge base are abstracted into rhetorical predicates which serve as sentential building blocks of text. Attachment points for linguistic units (partsof-speech, phrases, or complete utterances) are indicated by position in the rhetorical formalism. Prepositional phrase selection is guided by keywords such as function (for), location (in, on, under), or instrument (with, using).

\footnotetext{
${ }^{1}$ These distinguishing descriptive attributes, implicit in the expert system, were explicitly added to discriminate entities on the basis of structure, function, location, etc.
} 
The rhetorical formalism is interpreted with a case-frame semantics which is translated to syntactic form via a relational grammar. Discourse models of focus and context as well as rhetorical force guide syntax choices. Morphological synthesizers (using category and feature values from the syntax generator) together with orthographic routines govern final surface form (see figure 6). As illustrated in the final sentence of the paragraph, parenthetical functional justifications enhance the explanation by providing additional information from the knowledge base which was relevant but not included in the original explanation. levels of representation in EXPLAN can br viewed from this perspective.

Yet another area for further research concerns the replanning of explanations ir reaction to user feedback (Moore anc Swartout, 1988). Because of the explici representation of rhetorical structure, model: of discourse context (histories of foci rhetoric, and content), and alternativi explanation strategies, EXPLAN offers a ricl basis for investigating recovery strategie from a variety of explanation error states For example, input which indicates use misconception should guide the explanatior

\section{Why did the mission plan fail?}

Offensive Counter Air Mission 1002 has f-111e aircraft and a target of Ludwigslusts-Alpha. Ludwigslusts-Alpha is electronic hardware for early warning and ground counter interception. Therefore, the aircraft should be an $f-4 \mathrm{~g}$ (for radar destruction) rather than an $\mathrm{f}-111 \mathrm{e}$ (for radar suppression).

Figure 6. Rhetorically organized explanation of rule conflict.

\section{DISCUSSION}

The produced text is more effective because of explicit rhetorical organization, the use of textual connectives (e.g. "therefore"), and the enrichment of the explanation with additional justificatory knowledge. An interesting venue for further investigation, the order and dominance relationships of figure 4 could aid in responding to user misconceptions or follow-up questions. These relationships could be used to tailor rhetorical force to the type of user addressed, hence requiring explicit user models. An obvious weakness is the lack of goal-directed selection of rhetorical devices to achieve some targeted effect. In essence, pragmatic function is implicit in the rhetorical strategies such that effects on the hearer are achieved, although not explicitly planned for. A particularly enticing idea is that put forward by (Hovy, 1988) suggesting the need for both prescriptive, top-down planning of rhetorical goals, coupled with selectional restrictions at the surface level. Indeed, the planned rhetorical and constrained realization system to be more concrete, such a providing specific examples. Alternatively feedback which indicates that the use expertly follows the line of reasoning ma: suggest that the explanation strategy shouli minimize details or provide more abstrac reasoning. As a consequence, a flexibl explanation generator must be able to selec from multiple views of the underlyin: knowledge, such as structural versu functional representations (Suthers, 1988). I summary, the ability to provide justificatio dynamically using a range of explanatio strategies will greatly enhance the perspicuit and utility of complex knowledge base systems.

\section{CONCLUSION}

The EXPLAN system demonstrates th effectiveness of rhetorical organization textual connectives, and justificator enhancement of explanation traces to achiev more cohesive text. A more effectiv 
explanation/generation system will use knowledge about the user to select rhetorical structure, content, and surface choices and will be flexible enough to handle a variety of follow-up questions. These are the foci of current research.

\section{ACKNOWLEDGMENTS}

I would like to thank Professor Karen Sparck Jones for many enlightening discussions on issues concerning explanation and natural language generation.

\section{REFERENCES}

Appelt, Douglas. 1985 Planning English Sentences. Cambridge University Press, Cambridge, England.

Dawson, Bruce; Brown, Richard; Kalish, Candice and Goldkind, Stuart. 1987 Knowledge-based Replanning System. RADC Final Technical Report 87-60.

Grosz, Barbara and Sidner, Candice. 1988 Plans for Discourse. Harvard University TR-11-87. Also in Cohen, Paul; Morgan., J. and Pollack, Martha (eds.). 1988 Intentions in Communication, MIT Press.

Hovy, Eduard. 1988 Planning Coherent Multisentential Text. Proceedings of the 26th Annual Meeting of the Association of Computational Linguistics, Buffalo, NY.

Maybury, Mark. 1988a GENNY: A Knowledge Based Text Generation System. Proceedings of the RIAO Conference on User-Oriented ContentBased Text and Image Handling, MIT, Cambridge, MA: 849-862.

Maybury, Mark. 1988b Explanation Rhetoric: the Rhetorical Progression of Justifications. Proceedings of the AAAI-88 Workshop on Explanation, Minneapolis-St Paul, MN: 16-20.

McCoy, Kathy. 1985 Correcting ObjectRelated Misconceptions. Ph D Thesis, University of Pennsylvania TR 85-57.
McKeown, Kathy. 1985 Text Generation. Cambridge University Press, Cambridge, England.

Moore, Johanna and William Swartout. 1988 A Reactive Approach to Explanation. Proceedings of the Fouth International Workshop on Natural Language Generation.

Paris, Cecile Michael Wick, and William Thompson. 1988 The Line of Reasoning Versus the Line of Explanation. Proceedings of the AAAI-88 Workshop on Explanation, Minneapolis-St Paul, MN: 4-7.

Roberts, R. Bruce and Ira Goldstein. 1977 The FRL Manual. MIT AI Lab Memo 409.

Schank, Roger. 1986 Explanation Patterns: Understanding Mechanically and Creatively. Lawrence Erlbaum, Hillsdale, New Jersey.

Sidner, Candice. 1983 Focusing in the Comprehension of Definite Anaphora. In Brady and Berwick (eds.), Computational Models of Discourse. MIT Press, Cambridge, MA: 267-330.

Stevens, Albert and Cindy Steinberg. 1981 A Typology of Explanation and its Application to Intelligent Computer Aided Instruction. BBN Technical Report \#4626.

Suthers, Dan. 1988 Providing Multiple Views for Explanation. Proceedings of the AAAI-88 Workshop on Explanation, Minneapolis-St Paul, MN: 12-15.

Swartout, William. 1981 Producing Explanations and Justifications of Expert Consulting Programs. Ph D Thesis, MIT Tech Report No 251.

Swartout, William and Stephen Smoliar. 1988 Explaining the Link Between Causal Reasoning and Expert Behavior. in Topics in Medical and Artificial Intelligence, Miller. P. L. (ed.) SpringerVerlag. 